A Prototype English-Japanese Machine Translation System for Translating IBM Computer Manuals

Taijiro Tsutsumi

Natura1 Language Processing

Sctence Institute, IBM Japan, Ltd.

5-19, Sanban-cho, Chiyoda-ku

Tokyo 102, Japan

ABSTRACT

This paper describes a prototype English-Japanese machine translation (MT) system developed at the Science Institute of IBM Japan, Ltd. This MT system currently aims at the translation of IBM computer manuals. It is based on a transfer approach in which the transfer phase is divided into two sub-phases: English transformation and English-Japanese conversion. An outline of the system and a detailed description of the Eng1ish-Japanese transfer method are presented.

1. Introduction

The Science Institute of IBM Japan, Ltd. has been involved in English-Japanese machine translation for four years (1). We have developed a prototype capable of translating IBM computer manuals into Japanese. This system is based on a transfer approach in which the transfer process consists of English transformation and English-Japanese conversion. This MT system aims at 1) high-quality translation; 2) an easily maintained transfer component; and 3) a smaller Eng1ish-Japanese terminology dfctionary. The transformation rules and the conversion rules are presently being constructed through tests using the IBM manual "VM/SP General Information" (60P).

We are focusing on translation of IBM computer manuals for 3 reasons: 1) high-quality translation is expected in a 1 imited area; 2) English IBM manuals are presumably written as clearly as possible according to an IBM internal standard; 3) we already had a practical Eng1ish-Japanese terminology dictionary for human trans lators.

Most MT systems developed in Europe and the U.S. deal with language palrs in the Indo-European language group (2). In the case of English-Japanese translation, since both languages are categorized in different language groups, a more powerful linguist $1 c$ mechanism must be implemented. For instance, word order and sentence style are different and moreover an English word sometimes corresponds to more than one Japanese equivalent. To overcome these difficulties, an English-Japanese or Japanese-English $M T$ system might be based on a transfer or interlingua approach with a wide range of tree-transducing capabilities and a semantic processing mechanism.

\section{Overview of the system}

Fig. 1 shows the overali translation process. First of al1, an English sentence is syntactically analysed in the English analysis phase. The output of this analysis is one or more English parse trees. Second, in the English-Japanese transfer phase, an English parse tree, or an English intermediate representation, is transferred to a corresponding Japanese tree, or a Japanese intermediate representation. During this transfer, an English parse tree is at first transformed by the transformation component to an English tree in Japanese-like style, and this result is converted to a Japanese tree by the Engl1sh-Japanese conversion component.

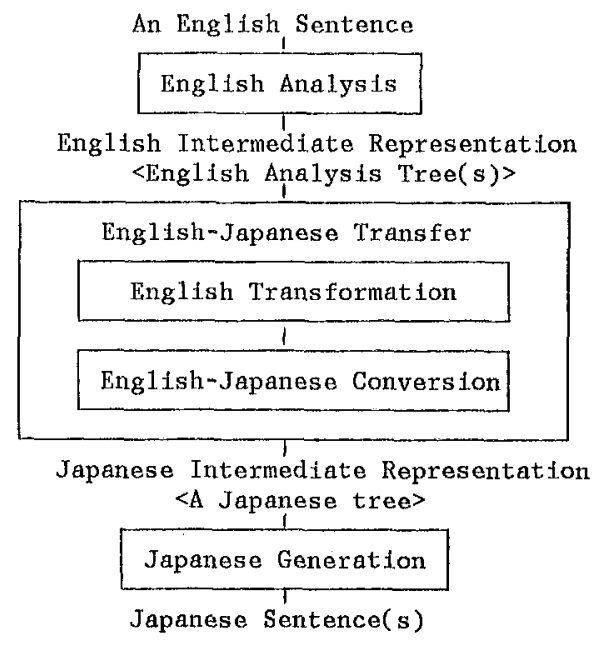

F1g. 1. Overa11 translation process

Finally, In the Japanese generation phase, one or more Japanese sentences are produced by operations such as generating Japanese auxilfary verbs, determining Japanese case particles, and rearranging word order. At present, the components shown in Fig. 1 are all implemented in trISP.

\section{English Analys is}

For analysing English, we are making use of the English parser, the English analysis grammar, and the Englfsh analysis dictionary developed by G. Heldorn et al. at the IBM T. J. Watson Research Center (3). The English analysts is based on an augmented phrase structure grammar and is syntactically performed in a bottom-up and parallel manner. This English analysis aims at area-independent, high-performance and fatl-soft analysis. The area-independent feature means portability of this analysis component to other application areas. The fail-soft feature is important for a practical MT system which should provide some Japanese segments for a human translator even if the parser falls to analyze the input sentence as a complete sentence.

As the syntactic analysis of English sometimes produces more than one parse tree, the English parser computes metric values which indicate plausibility of the parse trees based on the characteristics of the modifications between phrases (4). When more than one parse tree is obtained by analysis, semantically incorrect parse trees are discarded during the English-Japanese transfer. If more than one Japanese tree remains after the transfer, the metric values copied from these English parse trees to corresponding Japanese trees are used to rank these Japanese trees in terms of plausibility. The Japanese tree which has the least value, namely the most plaustble one, is chosen by the MT system. 
4. Eng1lish-Japanese Transfer

Generally, the transfer process of a transfer approach including semantic processing tends to become complicated and then difficult to maintain. But a transfex approach seems to be the most straightforward for implementing human translators' knowledge which includes vartious types of lingutstic information such as spectfic words, syntactic structures, and semantic information.

There are many Engltsh-proper expressions, such as 'It-that', '100-to', and 'there-be'. Their styles are very different from Japanese ones and have no simple contrast expressions in Japanese. 'The Fing1ish-Japanese transfer: component of our system is divided into two scparate components: an English transformation conponent and an English-Japanese conversion component. We call our approach a two-pass transfer method. By using English transformation rules, the ling 1 ish txans formation component rewrites an Finglish parse tree and produces a new style of English tree which is close to Japanese syntax. This can then ensily be converted to a corresponding Japanese tree. When we expect different English expresstons to be transiated to the same Japanese expcesston, we only have to wrtte linglish transformation rules instead of. $\mathrm{E}-\mathrm{J}$ trans fer rules of a conventional transfer approach. Moreover, when we have a Mr system change a Japanese expressiton, we are required only to modify some $\mathrm{E}-\mathrm{J}$ conversion rules instead of modifying a larger number of relating $E^{-} \mathrm{J}$ transfex rules. Consequently, the two pass transfer method provides us with modularity and maintainability of the transfor component.

\section{1 Englitsh Transformation}

English transformation is performed by using Fnglish transformation rules and a transformation dfctionary. The transformation sometimes requires a derfvative form of an English word, such as a verbal form of a noun and an adverbial form of an adjective. The transformation dictionary contains this sort of derivational data. The transformation rules are categorized into groups according to syntactic categories of nodes of parse trees. Each group is also classtfled Into several sub-groups. For example, the rule group for a sentence consists of 22 sub-groups, such as an inversion-rule sub-group, an insertion-rule sub-group, and an ellipsis-rule sub-group. The following are examples of applications of the rules to sentences.

It is required that you specify the assignment. $\rightarrow$ That you specify the assignment is required.

There are several records in the file.

$\rightarrow$ Several records exist in the file.

System operation is so impaired that the IPL procedure has to be repeated.

$\rightarrow$ Because system operation fs very impaired, the IPI, procedure has to be repeated.

The routine has a relatively low usage rate. $\rightarrow$ Usage tate of the routine is relatively low.

The following are examples of applications of the rules to noun phrases.

execution of the program

$\rightarrow$ executing the program

a disk avallable with ...

$\rightarrow$ a disk which is available with ...

one type of: ...

$\rightarrow$ one-type-of $\ldots$
The transformation is performed in a top-down manner along an Fnglish parse tree. At cach node of a tree, a corresponding rule group is retrieved according to the syntactic type of the node and this rute group is applied to tho sub-tree only once. In this app1ication of the rule group, each sub-group ts sequentially appli.ed to the sub-tree only once. If a matching pattern of a transformation rula matches the sub-tree and a target pattern produces a new tree, the rest of the rujes In the sub group are no longer used and procesising of the next sub-group begins. We have designed tho rule groups atul their sub-groups to avoid backtracking and repetitive application of the same rule.

4.2. English-Japanese Conyorsion

A transformed Fing1ish tree is convorted to a corre sponding Japanese tree by using conversion rules and a converston dictionary. The functions of thits process are 1.) determining appropriate Japanese syntax, equivalents, and additional 1.inguistic data such as tense, aspect, modality, and voice; and 2) disambiguating modiffeations of knglish phrises.

\subsubsection{Semantic Markers}

One of the basic approaches to semantic processing in MT is to use semantic markers of nouns. Wo have defined 24 semantic markers specific to computer manuals, which wi11 be effective in trans 1ating TBM computer maniluals. Table 1 lists all of the semantic markers and their meantugs.

\begin{tabular}{|c|c|c|c|}
\hline $\begin{array}{l}\text { Semantic } \\
\text { Markors }\end{array}$ & Meanings & $\begin{array}{l}\text { Semant } d c \\
\text { Markors }\end{array}$ & Meanings \\
\hline $\mathrm{I}, \mathrm{C}$ & Logical Containec & WK & Work/Action \\
\hline IE & Logica1 Entry & PS & Predicate \\
\hline $\mathrm{LP}$ & Logical Path & $\mathrm{AP}$ & Attribute of PS \\
\hline DM & Document & SL & Supply \\
\hline $\mathrm{ST}$ & State & PT & Part \\
\hline $\mathrm{TH}$ & Technique/Theory & DT' & Term of documents \\
\hline FA & Feature/Ability & ML & Material \\
\hline IF & Information & TM & Time \\
\hline $\mathrm{AT}$ & Attribute & PI, & Place \\
\hline VA & Value of AT & PN & Person's Name \\
\hline HM & Human & po & Point \\
\hline UD & Unit/Device & OG & Organization \\
\hline
\end{tabular}

Table 1. Semantic markers

Nouns in computer manuals have one or more semantic markers. For example, "ffle" has "LC" and "LE", "program" has "LE", and "operator" has "LE" and "HM". This set of markers is so simple that maintenance is easy.

4.2.2. E-J Conversion Dictionary

In the English-Japanese converston dictionary, conditions for converston are described by a combination of English syntax, semantic markers and sometimes spectific Japanese words. The converston dictionary is divided into sub-dictionaries, such as a verb-dictionary, a noun-dictionary, and a prepositional-dictionary. Fig. 2 shows an example of an entry of the verb-dictlonary in the case of "provide".

$$
\begin{aligned}
& \text { ("provide" } \\
& \text { (( } \mathrm{SB} \text { (S (( IE UD) Y1 "ga" )) ) } \\
& \text { (DO (S ((FA AT) Y1 "wo"))) } \\
& \text { ( } \mathrm{P} \text { "sonae" PY1 (V SIITMO1 NII JYOOTAI TRANS)) ) } \\
& \text { ((SB (S ((DM LE UD) } \mathrm{Y}_{1} \text { "ga"))) } \\
& \text { (DO (S ((HM) Y1 "nf"))) } \\
& \text { ("witb" ( S (( IF FA AT) Y1 "wo"))) } \\
& \text { ( } P \text { "teikyo" PY1 (V SAHEN NIL KEIZOKU TRANS })) \text { ) ) }
\end{aligned}
$$

Fig. 2. An example of an Einglish-Japanese conversion dfctionaxy entry 
The upper half of the description in Fig. 2 specifies that if the subject of a sentence has semantic marker "LE" or "UD" and the first object has marker "FA" or "Al", then choose the Japanese case particle "ga" for the first Japanese noun phrase, the Japanese case particle "wo" for the second one, and the Japanese verb "sonae" as the proper equivalent for the English verb "provide". "Y1" and "PY1" in Fig. 2 specify types of corresponding Japanese sub-trees to be generated. The lower half of the description gives a similar rule to the previous one except for an additional condition on a prepositional phrase. This part specifies that if the conditions are met, then use Japanese case particles "ga", "ni", and "wo" in this order and select "tetkyo" as the appropriate Japanese verb.

The verb-dictionary is used to convert an English surface case structure into a Japanese one directly by depending upon the semantic markers. This conversion must be more efficient than in the case where deep cases are introduced so as to pursue similar semantic processing. This conversion determines an appropriate Japanese verb, Japanese case particles, and Japanese syntax of a simple sentence at the same time. In some cases, an appropriate Japanese equivalent for an English noun phrase is successfully selected based on these conditions when the English noun phrase has more than one Japanese equivalent. Moreover, applifcation of these entries also means a semantic check of the input from the computer area's point of view. Consequently, If there is no entry applicable to the input simple sentence, it is deemed inappropriate for computer manuals and is rejected by the system. This contributes to disambiguation of English analysis trees.

Additional 1inguistic data of an English simple sentence concerning tense, aspect, modality, and voice, are also converted to corresponding data of a Japanese tree by using a contrast conversion table and the conversion dictionary. For example, volce and aspect of an English sentence are changed in a Japanese sentence according to the characteristic of the verb.

4.2.3. E-J Translation of Simple Noun Phrases

One of the issues in MT is how to create and maintain a large terminology dictionary. Generally, a technical document includes a number of technical noun groups. We call a noun phrase whtch bastcally has no post modifier a simple noun phrase (SNP), such as "a procedure 1ibrary", "system-to-operator communication", "IBM supplied licensed and nonlicensed programs" and "page $34^{\prime \prime}$.

Our MT system facilitates a component for translating SNPs. Even if the terminology dictionary does not have the entry in whole, a long SNP which is composed of many words is successfully translated by appropriately assembling the dictionary data of all elements of the SNP. This is mainly due to the similarity of syntax of SNPs between English and Japanese.

The functions of the SNP translation component are to choose appropriate Japanese equivalents for various parts-of-speech(e.g. noun, adjective, adverb); to insert "no" between noun phrases; to reorder Japanese equivalents; to process conjunctions within a simple noun phrase; and to handle hyphenated words. These are achieved by using a special dictionary for translating SNPs and co-occurrence frequency data of words or semantic markers in IBM computer manuals.

4.3 E-I Conversion Process

The Englfsh-Japanese conversion component sabsequently converts a transformed English tree to a Japanese tree in a bottom-up and parallel manner along the English tree.

First of al1, the Eng1ish-Japanese conversion dictionary is searched for al1 English words which are terminal symbols of the English parse tree. This is part of Eng1/sh-Japanese conversion of the lowest leve1 sub-trees of the English tree. An upper level English sub-tree is converted to a corresponding Japanese sub-tree by using the English-Japanese conversion rules and by using the English-Japanese conversion results of the current level English sub-trees. The category of the top node of the upper sub-tree determines which set of English-Japanese conversion rules is to be applied. During the conversion of sub-trees, semantic processing is performed according to the data in the English-Japanese conversion dictionary as mentioned ear1ier.

\section{Japanese Generation}

The Japanese generation component produces one or more Japanese sentences from a Japanese tree which conveys Japanese syntax, Japanese equivalents, and other information.

The functions of this component are to generate Japanese auxiliary verbs; to determine appropriate Japanese equivalents of adverbs, negation, determiners and conjunctions including subordinate conjunctions; to position Japanese adverbial phrases in a Japanese sentence; to modify Japanese case particles; to reordex Japanese noun phrases; to tnsert punctuations; and to erase a duplicate Japanese subject. Japanese auxilfary verbs are generated based on Japanese verb information, such as the original form of the verb, the conjugation type of the verb, tense, aspect, voice, and modality.

6. Concluston

We have described a prototype English-Japanese machine translation system based on a two-pass transfer approach. Introduction of separate English transformation in the $\mathrm{E}-\mathrm{J}$ transfer makes the transfer component easy to maintain.

We have proposed a set of semantic markers specific to computer manua1s and the English-Japanese conversion dictlonary so as to perform high-quality translation. The mechanism of selecting appropriate Japanese equivalents and syntax is simple and effective. We will continue to enhance our MT system to translate many kinds of IBM computer manuals into high-quality Japanese.

7. References

1. Tsutsumi, T. "On the Machfne Translation from English to Japanese" in Tokyo Scientific Center Report N: G318-1571 (1982)

2. Slocum, J. "A Survey of Machine Translation: its History, Current Status, and Future Prospects" in AJCL 11-1 (1985)

3. Heidorn, G.E., K. Jensen, L.A. Miller, R. J. Byrd, and M.S. Chodorow. "The EPISTLE Text-Critiquing System" In IBM Sys. J. 21.3 (1982), 305-326.

4. Heidorn, G. E. "Experience with an Easily Computed Metric for Ranking Alternative Parses" in Proc. 20th Annual Meeting of the ACL. Tronto, Canada (1982), 82-84. 